\title{
ON THE FINITENESS OF BASS NUMBERS OF LOCAL COHOMOLOGY MODULES OVER NOETHERIAN REGULAR LOCAL RINGS
}

\author{
YAVAR IRANI \\ Received 16 October, 2015
}

\begin{abstract}
Let $R$ be a commutative Noetherian regular local ring containing a field. Let $I$ be an ideal of $R$ and let $\ell \geq 0$ be an integer. In this paper it is shown that for every finitely generated $R$-module $M$ and each integer $i \geq \ell$, the Bass numbers of the $R$-module $H_{I}^{i}(M)$ are finite, whenever, $\operatorname{dim} \operatorname{Supp}\left(H_{I}^{i}(R)\right) \leq 1$, for all $i \geq \ell$.
\end{abstract}

2010 Mathematics Subject Classification: 13D45; 14B15; 13E05

Keywords: Bass numbers, cominimax modules, local cohomology, regular ring

\section{INTRODUCTION}

Let $R$ denote a commutative Noetherian ring and let $I$ be an ideal of $R$. In [10], Hartshorne defined an $R$-module $L$ to be $I$-cofinite, if $\operatorname{Supp}(L) \subseteq V(I)$ and $\operatorname{Ext}_{R}^{i}(R / I, L)$ is finitely generated module for all $i$. He posed the following question:

Is the category $\mathscr{M}(R, I)_{\text {cof }}$ of I-cofinite modules forms an Abelian subcategory of the category of all $R$-modules? That is, if $f: M \longrightarrow N$ is an R-homomorphism of I-cofinite modules, are $\operatorname{ker} f$ and coker $f$ I-cofinite?

Hartshorne proved that if $I$ is a prime ideal of dimension one in a complete regular local ring $R$, then the answer to his question is yes. On the other hand, in [7], Delfino and Marley extended this result to arbitrary complete local rings. Kawasaki [13] generalized the Delfino and Marley's result for an arbitrary ideal $I$ of dimension one in a local ring $R$. Recently, Bahmanpour et al in [4] and Melkersson in [19] have removed the local assumption on $R$.

Recall that, we say that $M$ is a minimax module if there is a finitely generated submodule $N$ of $M$, such that $M / N$ is Artinian. The interesting class of minimax modules was introduced by H. Zöshinger in [20] and he has given in [20] and [21] many equivalent conditions for a module to be minimax. Also, the $R$-module $M$ is

This work of the author was supported by a grant from Meshkin-Shahr branch Islamic Azad University.

(c) 2016 Miskolc University Press 
said to be $I$-cominimax, if support of $M$ is contained in $V(I)$ and $\operatorname{Ext}_{R}^{i}(R / I, M)$ is minimax for all $i \geq 0$. The concept of the $I$-cominimax modules were introduced in [2] as a generalization of important notion of $I$-cofinite modules.

In this paper as a generalization the main results of [4] and [19] to the class of cominimax modules we prove the following:

Let $(R, \mathfrak{m})$ be a Noetherian local ring and let I be an ideal of $R$ of dimension one. Let $\mathscr{M}(R, I)_{\text {com }}$ denote the category of I-cominimax modules. Then $\mathscr{M}(R, I)_{\text {com }}$ forms an Abelian subcategory of the category of all $R$-modules.

On the other hand, an important problem in commutative algebra is determining when the set of associated primes and the Bass numbers of the $i^{\text {th }}$ local cohomology module $H_{I}^{i}(M)$ are finite. Lyubeznik conjectured that,

If $R$ is a regular ring and $I$ is an ideal of $R$, then the local cohomology modules $H_{I}^{i}(R)$ have finitely many associated prime ideals for all $i \geq 0$, (see [14, Remark 3.7(iii)]).

This conjecture have solved with an affirmative answer by Huneke and Sharp [12] and Lyubeznik in [14] and [15] for regular rings containing a field. Also, in [11] Huneke conjectured that,

For any ideal $I$ in a regular local ring $(R, \mathfrak{m})$, the Bass numbers

$$
\mu^{j}\left(\mathfrak{p}, H_{I}^{i}(R)\right)=\operatorname{dim}_{k(\mathfrak{p})} \operatorname{Ext}_{R_{\mathfrak{p}}}^{j}\left(k(\mathfrak{p}), H_{I R_{\mathfrak{p}}}^{i}\left(R_{\mathfrak{p}}\right)\right)
$$

are finite for all $i$ and $j$, where $k(\mathfrak{p}):=R_{\mathfrak{p}} / \mathfrak{p} R_{\mathfrak{p}}$. In particular the injective resolution of the local cohomology has only finitely many copies of the injective hull of $R / \mathfrak{p}$ for any $\mathfrak{p}$.

Huneke and Sharp [12] and Lyubeznik [14,15] have shown that this conjecture holds for any regular local ring containing a field. But both of this conjectures are still open for the general case. Also, in some situations the second conjecture holds even for every finitely generated $R$-module instead of the ring $R$. (For example, see [1]).

The main purpose of this note is to prove the following:

Let $(R, \mathfrak{m})$ be a Noetherian regular local ring containing a field and let $I$ be an ideal of $R$. Let $\ell \geq 0$ be an integer such that $\operatorname{dim} \operatorname{Supp}\left(H_{I}^{i}(R)\right) \leq 1$, for each $i \geq \ell$. Then for every finitely generated $R$-module $M$ and for each integer $i \geq \ell$, the set $\operatorname{Supp}\left(H_{I}^{i}(M)\right)$ is finite and the Bass numbers of the R-module $H_{I}^{i}(M)$ are finite.

Throughout this paper, $R$ will always be a commutative Noetherian ring with nonzero identity and $I$ will be an ideal of $R$. For any $R$-module $M$, the $i^{t h}$ local cohomology module of $M$ with support in $I$ is defined by

$$
H_{I}^{i}(M)=\underset{n \geq 1}{\lim } \operatorname{Ext}_{R}^{i}\left(R / I^{n}, M\right) .
$$

We refer the reader to [9] or [6] for the basic properties of local cohomology.

For any ideal $\mathfrak{a}$ of $R$, we denote $\{\mathfrak{p} \in \operatorname{Spec} R: \mathfrak{p} \supseteq \mathfrak{a}\}$ by $V(\mathfrak{a})$. For any unexplained notation and terminology we refer the reader to [6] and [17]. 


\section{Finiteness of BASS NUMBERS OF LOCAL COHOMOLOGY MODULES}

The following well known lemmata are crucial for the proof of Theorem 1.

Lemma 1. Let $(R, \mathfrak{m})$ be a Noetherian local ring and let $I$ be an ideal of $R$ with $\operatorname{dim} R / I=1$. Assume that $M$ is an $R$-module and $n \geq 0$ is an integer. Then the following conditions are equivalent:

(1) $\mu^{i}(\mathfrak{p}, M)$ is finite for all $\mathfrak{p} \in V(I)$ and for all $0 \leq i \leq n$;

(2) The $R$-module $\operatorname{Ext}_{R}^{i}(R / I, M)$ is minimax, for all integers $0 \leq i \leq n$.

Proof. See [5, Theorem 2.3].

Recall that for an $R$-module $M$, the cohomological dimension of $M$ with respect to ideal $I$ of $R$, is defined as

$$
\operatorname{cd}(I, M):=\max \left\{i \in \mathbb{Z}: H_{I}^{i}(M) \neq 0\right\} .
$$

Lemma 2. Let $R$ be a Noetherian ring and let $M$ be an $R$-module of finite dimension $d$. Then the followings are equivalent:

(1) For each $\mathfrak{p} \in \operatorname{Spec}(R)$ and any integer $0 \leq i \leq d$, the Bass numbers $\mu^{i}(\mathfrak{p}, M)$ are finite;

(2) For each $\mathfrak{p} \in \operatorname{Spec}(R)$ and any integer $i \geq 0$, the Bass numbers $\mu^{i}(\mathfrak{p}, M)$ are finite.

Proof. (2) $\Rightarrow$ (1) Is clear.

$(1) \Rightarrow(2)$ Using localization we may assume $(R, \mathfrak{m}, k)$ is a local Noetherian ring and $\mathfrak{p}=\mathfrak{m}$ is the unique maximal ideal of $R$. As by [6, Corollary 10.2.8] the $R$ module $E_{R}(k)$, the injective hull of $k$, is Artinian it follows from the hypothesis and from the definition of local cohomology modules that for any $0 \leq i \leq d$ the $R$-module $H_{\mathfrak{m}}^{i}(M)$ is Artinian. But in view of [6, Theorem 6.1.2] we have $H_{\mathfrak{m}}^{i}(M)=0$ for all integers $i>d$. Therefore for all integers $i \geq 0$ the $R$-modules $H_{\mathfrak{m}}^{i}(M)$ are Artinian and hence are $\mathfrak{m}$-cofinite. So the assertion follows from the [18, Proposition 3.9].

Lemma 3. Let $R$ be a Noetherian ring and let $\ell_{B}^{1}(R)$ denote the category of all $R$-modules $M$ with $\operatorname{dim} \operatorname{Supp}(M) \leq 1$ such that all Bass numbers of $M$ are finite. Then $\bigcup_{B}^{1}(R)$ forms an Abelian subcategory of the category of all $R$-modules.

Proof. Let $M, N \in \ell_{B}^{1}(R)$ and let $f: M \rightarrow N$ be an $R$-homomorphism. It is enough to prove that the $R$-modules $\operatorname{ker}(f)$ and coker $(f)$ are in $\ell_{B}^{1}(R)$.

Now, the exact sequence

$$
0 \longrightarrow \operatorname{ker}(f) \longrightarrow M \longrightarrow \operatorname{im}(f) \longrightarrow 0
$$

implies that for each $\mathfrak{p} \in \operatorname{Spec}(R)$ and any $0 \leq i \leq 1$ the Bass numbers $\mu_{R}^{i}(\mathfrak{p}, \operatorname{ker}(f))$ are finite. So, by Lemma 2 it follows that $\operatorname{ker}(f)$ is in $\mathcal{C}_{B}^{1}(R)$. Now the reminder section of the proof follows from the exact sequences

$$
0 \longrightarrow \operatorname{ker}(f) \longrightarrow M \longrightarrow \operatorname{im}(f) \longrightarrow 0,
$$


and

$$
0 \longrightarrow \operatorname{im}(f) \longrightarrow N \longrightarrow \operatorname{coker}(f) \longrightarrow 0 .
$$

Now, we are ready to state and prove our first main result.

Theorem 1. Let $(R, \mathfrak{m})$ be a Noetherian local ring and let $I$ be an ideal of $R$ with $\operatorname{dim} R / I=1$. Let $\mathscr{M}(R, I)_{\text {com }}$ denote the category of $I$-cominimax modules over $R$. Then $\mathscr{M}(R, I)_{\text {com }}$ forms an Abelian subcategory of the category of all $R$-modules.

Proof. The assertion follows from Lemma 1 and 3.

The following lemma plays a key role in the proof of Proposition 1.

Lemma 4. Let $(R, \mathfrak{m})$ be a Noetherian local ring and let I be an ideal of $R$. Let $M$ be a finitely generated $R$-module of dimension $d$. Let $\ell \geq 0$ be an integer such that $\operatorname{cd}(I, M) \geq \ell$. Assume that, for each $i \geq \ell$, the set $\operatorname{Supp}\left(H_{I}^{i}(M)\right)$ is finite and the Bass numbers of the $R$-module $H_{I}^{i}(M)$ are finite. Then, for each $i \geq \ell$, the $R$-module $H_{I}^{i}(M)$ is $J$-cominimax, where,

$$
J:=\bigcap_{\mathfrak{p} \in \operatorname{Supp}\left(\oplus_{i \geq \ell} H_{I}^{i}(M)\right)} \mathfrak{p} .
$$

Proof. From the hypothesis $\operatorname{cd}(I, M) \geq \ell$ it follows that $\operatorname{Supp}\left(\oplus_{i \geq \ell} H_{I}^{i}(M)\right) \neq$ $\varnothing$. Also since by the hypothesis, for each $\ell \geq 0$, the set $\operatorname{Supp}\left(H_{I}^{i}(M)\right)$ is finite and by Grothendiek's Vanishing Theorem, for every $i>d$, we have $H_{I}^{i}(M)=0$, it follows that the set $\operatorname{Supp}\left(\oplus_{i \geq \ell} H_{I}^{i}(M)\right)$ is finite and hence $\operatorname{dim}(R / J) \leq 1$. Now if $\operatorname{dim}(R / J)=0$, then we have $J=\mathfrak{m}$ and so the assertion is clear. So, we may assume $\operatorname{dim}(R / J)=1$. But in this situation the assertion follows immediately from Lemma 1. (Note that $\operatorname{Supp}\left(H_{I}^{i}(M)\right) \subseteq V(J)$, for each $i \geq \ell$ ).

The following lemma and its corollary is needed in the proof of Proposition 1.

Lemma 5. Let $R$ be a Noetherian ring and let $I$ be an ideal of $R$. Let $M$ and $N$ be two finitely generated $R$-modules and let $\ell \geq 0$ be an integer. If $\operatorname{Supp}(N) \subseteq$ $\operatorname{Supp}(M)$, then we have

$$
\bigcup_{i \geq \ell} \operatorname{Supp}\left(H_{I}^{i}(N)\right) \subseteq \bigcup_{i \geq \ell} \operatorname{Supp}\left(H_{I}^{i}(M)\right)
$$

Proof. Let $\mathfrak{p} \in \cup_{i \geq \ell} \operatorname{Supp}\left(H_{I}^{i}(N)\right)$. Then there exists an integer $j \geq \ell$ such that $\left(H_{I}^{j}(N)\right)_{\mathfrak{p}} \neq 0$, which implies that $H_{I R_{\mathfrak{p}}}^{j}\left(N_{\mathfrak{p}}\right) \neq 0$. Therefore, it follows from the definition that $\operatorname{cd}\left(I R_{\mathfrak{p}}, N_{\mathfrak{p}}\right) \geq j$. Now as $\operatorname{Supp}\left(N_{\mathfrak{p}}\right) \subseteq \operatorname{Supp}\left(M_{\mathfrak{p}}\right)$ it follows from [8, Theorem 2.2], that $\operatorname{cd}\left(I R_{\mathfrak{p}}, M_{\mathfrak{p}}\right) \geq \operatorname{cd}\left(I R_{\mathfrak{p}}, N_{\mathfrak{p}}\right) \geq j \geq \ell$. So, if we have $\operatorname{cd}\left(I R_{\mathfrak{p}}, M_{\mathfrak{p}}\right)=t$, then we have $H_{I R_{\mathfrak{p}}}^{t}\left(M_{\mathfrak{p}}\right) \neq 0$. Therefore, $\left(H_{I}^{t}(M)\right)_{\mathfrak{p}} \neq 0$ and hence $\mathfrak{p} \in \cup_{i \geq \ell} \operatorname{Supp}\left(H_{I}^{i}(M)\right)$. 
Corollary 1. Let $R$ be a Noetherian ring and let $I$ be an ideal of $R$. Let $M a$ finitely generated $R$-modules and $\ell \geq 0$ be an integer. Then

$$
\bigcup_{i \geq \ell} \operatorname{Supp}\left(H_{I}^{i}(M)\right) \subseteq \bigcup_{i \geq \ell} \operatorname{Supp}\left(H_{I}^{i}(R)\right)
$$

Proof. Since $\operatorname{Supp}(M) \subseteq \operatorname{Spec}(R)=\operatorname{Supp}(R)$, the assertion follows immediately from Lemma 5.

The following proposition is crucial for the proof of Theorem 2.

Proposition 1. Let $(R, \mathfrak{m})$ be a Noetherian local ring and let $I$ be an ideal of $R$. Let $\ell \leq \operatorname{cd}(I, R)$ be an integer such that for each $i \geq \ell$, the set $\operatorname{Supp}\left(H_{I}^{i}(R)\right)$ is finite and the Bass numbers of the $R$-module $H_{I}^{i}(R)$ are finite. Then the following statements hold:

(1) For every finitely generated $R$-module $M$ with finite projective dimension and each integer $i \geq \ell$, the $R$-module $H_{I}^{i}(M)$ is $J$-cominimax, where,

$$
J:=\bigcap_{\mathfrak{p} \in \operatorname{Supp}\left(\oplus_{i \geq \ell} H_{I}^{i}(R)\right)} \mathfrak{p} .
$$

(2) For every finitely generated $R$-module $M$ with finite projective dimension and for each integer $i \geq \ell$, the set $\operatorname{Supp}\left(H_{I}^{i}(M)\right)$ is finite and the Bass numbers of the R-module $H_{I}^{i}(M)$ are finite.

Proof. (1) For every finitely generated $R$-module $M$, by the Grothendiek's Vanishing Theorem we have $H_{I}^{i}(M)=0$, for each integer $i>\operatorname{dim}(R)$ and so we have $\ell \leq \operatorname{dim}(R)$. Now, we argue by induction on $t:=\operatorname{dim}(R)-\ell$. If $t=0$, then for every finitely generated $R$-module $M$ (even not necessary with finite projective dimension), using Grothendiek's Vanishing Theorem and [18, Proposition 5.1], it is straightforward to see that, the $R$-module $H_{I}^{\operatorname{dim}(R)}(M)$ is $I$-cofinite and hence is $I$-cominimax. Now, if $H_{I}^{\operatorname{dim}(R)}(M) \neq 0$, then the assertion follows from Corollary 1 and [2, Corollary 2.8]. (Note that in this situation we have $\{\mathfrak{m}\}=\operatorname{Supp}\left(H_{I}^{\operatorname{dim}(R)}(M)\right)=V(J) \subseteq$ $V(I)$ ). Now, let $t>0$ and inductively, the assertion has been proved for all smaller values of $t$ for all finitely generated $R$-modules with finite projective dimension. Then by inductive hypothesis, for every finitely generated $R$-module $M$ with finite projective dimension and each $i \geq \ell+1$, the $R$-module $H_{I}^{i}(M)$ is $J_{1}$-cominimax, where,

$$
J_{1}:=\bigcap_{\mathfrak{p} \in \operatorname{Supp}\left(\oplus_{i \geq \ell+1} H_{I}^{i}(R)\right)} \mathfrak{p} .
$$

Then as by the hypothesis the set $\operatorname{Supp}\left(H_{I}^{\ell}(R)\right)$ is finite, it follows that $\operatorname{dim}(R / \mathfrak{q}) \leq 1$ for every $\mathfrak{q} \in \operatorname{Supp}\left(H_{I}^{\ell}(R)\right)$ and so using Lemma 1 , it follows that, for every finitely 
generated $R$-module $M$ with finite projective dimension and each $i \geq \ell+1$, the $R$ module $H_{I}^{i}(M)$ is $J$-cominimax, where,

$$
J:=\bigcap_{\mathfrak{p} \in \operatorname{Supp}\left(\oplus_{i \geq \ell} H_{I}^{i}(R)\right)} \mathfrak{p} .
$$

(Note that $\operatorname{dim}(R / J) \leq 1)$. Next, let $M$ be an arbitrary non-zero finitely generated $R$ module with finite projective dimension. Then, we argue on $s:=\operatorname{projdim}_{R}(M)$ that, the $R$-module $H_{I}^{\ell}(M)$ is $J$-cominimax. For $s=0$, the assertion follows from Lemma 4. Now let $s>0$ and the result has been proved for all finitely generated $R$-modules with finite projective dimension smaller than $s$. Let $M$ be a finitely generated $R$ -

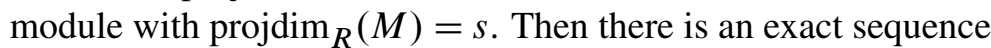

$$
0 \rightarrow K \rightarrow F \rightarrow M \rightarrow 0
$$

for some finitely generated free $R$-modules $F$ and some finitely generated $R$-modules $K$ with $\operatorname{projdim}_{R}(K)=s-1$. Then by inductive hypothesis of the second inductive argument, the $R$-modules $H_{I}^{\ell}(K)$ and $H_{I}^{\ell}(F)$ are $J$-cominimax. Moreover, by the inductive hypothesis of the first inductive argument, the $R$-modules $H_{I}^{\ell+1}(K)$ and $H_{I}^{\ell+1}(F)$ are $J$-cominimax. On the other hand, the exact sequence 2.1 induces the following exact sequence

$$
H_{I}^{\ell}(K) \stackrel{f}{\rightarrow} H_{I}^{\ell}(F) \rightarrow H_{I}^{\ell}(M) \rightarrow H_{I}^{\ell+1}(K) \stackrel{g}{\rightarrow} H_{I}^{\ell+1}(F) .
$$

Now the exact sequence 2.2 yields the exact sequence

$$
0 \rightarrow \operatorname{Coker}(f) \rightarrow H_{I}^{\ell}(M) \rightarrow \operatorname{Ker}(g) \rightarrow 0 .
$$

On the other hand, the exact sequence 2.3 induces the exact sequence

$$
\begin{aligned}
& 0 \rightarrow \operatorname{Hom}_{R}(R / J, \operatorname{Coker}(f)) \rightarrow \operatorname{Hom}_{R}\left(R / J, H_{I}^{\ell}(M)\right) \rightarrow \operatorname{Hom}_{R}(R / J, \operatorname{Ker}(g)) \\
& \rightarrow \operatorname{Ext}_{R}^{1}(R / J, \operatorname{Coker}(f)) \rightarrow \operatorname{Ext}_{R}^{1}\left(R / J, H_{I}^{\ell}(M)\right) \rightarrow \operatorname{Ext}_{R}^{1}(R / J, \operatorname{Ker}(g)) \rightarrow \cdots,
\end{aligned}
$$

which using [3, Lemma 2.1] and Theorem 1, implies that the $R$-module $H_{I}^{\ell}(M)$ is $J$-cominimax. This completes the second inductive step. Now the inductive step of the first inductive argument is complete, too.

(2) The assertion follows immediately from (i), using Lemma 1 and Corollary 1.

Now we are ready to state and prove our second main result.

Theorem 2. Let $(R, \mathfrak{m})$ be a Noetherian local regular ring containing a field and let $I$ be an ideal of $R$. Let $0 \leq \ell \leq \operatorname{cd}(I, R)$ be an integer such that $\operatorname{dim} \operatorname{Supp}\left(H_{I}^{i}(R)\right) \leq$ 1 , for each $i \geq \ell$. Then the following statements hold: 
(1) For every finitely generated $R$-module $M$ and each integer $i \geq \ell$, the $R$ module $H_{I}^{i}(M)$ is $J$-cominimax, where,

$$
J:=\bigcap_{\mathfrak{p} \in \operatorname{Supp}\left(\oplus_{i \geq \ell} H_{I}^{i}(R)\right)} \mathfrak{p} .
$$

(2) For every finitely generated $R$-module $M$ and for each integer $i \geq \ell$, the set $\operatorname{Supp}\left(H_{I}^{i}(M)\right)$ is finite and the Bass numbers of the $R$-module $H_{I}^{i}(M)$ are finite.

Proof. In view of [12] and [14,15], the Bass numbers of the $R$-module $H_{I}^{i}(R)$ are finite, for all $i$. Moreover, in view of [12] and [14,16], the set $\operatorname{Ass}_{R}\left(H_{I}^{i}(R)\right)$ is finite, for all $i$. In particular, if $\operatorname{dim} \operatorname{Supp}\left(H_{I}^{i}(R)\right) \leq 1$ then the set $\operatorname{Supp}\left(H_{I}^{i}(R)\right)$ is finite. Now, as $(R, \mathfrak{m})$ is a regular ring, it follows that any finitely generated $R$-module has finite projective dimension. Now, we can conclude the assertion immediately from Proposition 1.

Corollary 2. Let $(R, \mathfrak{m})$ be a Noetherian regular local ring of dimension $d \geq 1$, containing a field. Then for any finitely generated $R$-module $M$, the Bass numbers of the local cohomology module $H_{I}^{i}(M)$ are finite, for all integers $i \geq d-1$.

Proof. In view of the main results of [12], [14] and [15], the set $\operatorname{Ass}_{R}\left(H_{I}^{i}(R)\right)$ is finite, for all integers $i \geq d-1$. Also, in view of Grothendiek's Vanishing Theorem we have $\operatorname{dim} \operatorname{Supp}\left(H_{I}^{i}(R)\right) \leq 1$, for all integers $i \geq d-1$. Now, it is clear that $\operatorname{dim} \operatorname{Supp}\left(H_{I}^{i}(R)\right) \leq 1$, for each $i \geq d-1$ and hence the assertion follows from Theorem 2.

\section{ACKNOWLEDGMENTS}

The author is deeply grateful to the referee for a very careful reading of the manuscript and many valuable suggestions in improving the quality of the paper. Also, the author would like to thank the Islamic Azad University-Meshkin-Shahr Branch for its financial support.

\section{REFERENCES}

[1] N. Abazari and K. Bahmanpour, "On the finiteness of Bass numbers of local cohomology modules," J. Alg. Appl., vol. 10, no. 4, pp. 783-791, 2011, doi: 10.1142/S0219498811004926.

[2] J. Azami, R. Naghipour, and B. Vakili, "Finiteness properties of local cohomology modules for a-minimax modules," Proc. Amer. Math. Soc., vol. 137, no. 2, pp. 439-448, 2009, doi: 10.1090/S0002-9939-08-09530-0.

[3] K. Bahmanpour and R. Naghipour, "On the cofiniteness of local cohomology modules," Proc. Amer. Math. Soc., vol. 136, no. 7, pp. 2359-2363, 2008, doi: 10.1090/S0002-9939-08-09260-5.

[4] K. Bahmanpour, R. Naghipour, and M. Sedghi, "On the category of cofinite modules which is Abelian," Proc. Amer. Math. Soc., vol. 142, no. 4, pp. 1101-1107, 2014, doi: 10.1090/S00029939-2014-11836-3. 
[5] K. Bahmanpour, R. Naghipour, and M. Sedghi, "On the finiteness of Bass numbers of local cohomology modules and cominimaxness," Houston J. Math., vol. 40, no. 2, pp. 319-337, 2014.

[6] M. Brodmann and R. Sharp, Local cohomology; an algebraic introduction with geometric applications, ser. Cambridge Studies in Advanced Mathematics. Cambridge, UK: Cambridge University Press, 1998, vol. 60.

[7] D. Delfino and T. Marley, "Cofinite modules and local cohomology," J. Pure Appl. Algebra, vol. 121, no. 1, pp. 45-52, 1997, doi: 10.1016/S0022-4049(96)00044-8.

[8] K. Divaani-Aazar, R. Naghipour, and M. Tousi, "Cohomological dimension of certain algebraic varieties," Proc. Amer. Math. Soc., vol. 130, no. 12, pp. 3537-3544, 2002, doi: 10.1090/S00029939-02-06500-0.

[9] A. Grothendieck, Local cohomology, ser. Notes by R. Hartshorne, Lecture Notes in Math. Berlin - Heidelberg - New York: Springer-Verlag., 1967, vol. 41.

[10] R. Hartshorne, “Affine duality and cofiniteness," Invent. Math., vol. 9, no. 2, pp. 145-164, 1970, doi: 10.1007/BF01404554.

[11] C. Huneke, "Problems on local cohomology," Free resolutions in commutative algebra and algebraic geometry, (Sundance,Utah,1990), Research Notes in Mathematics, Boston, MA, Jones and Bartlett Publishers, vol. 2, pp. 93-108, 1994.

[12] C. Huneke and R. Sharp, "Bass numbers of local cohomology modules," Trans. Amer. Math. Soc., vol. 339, no. 2, pp. 765-779, 1993, doi: 10.1090/S0002-9947-1993-1124167-6.

[13] K.-I. Kawasaki, "On a category of cofinite modules which is Abelian," Math. Z., vol. 269, no. 1, pp. 587-608, 2011, doi: 10.1007/s00209-010-0751-0.

[14] G. Lyubeznik, "Finiteness properties of local cohomology modules (an application of Dmodules to commutative algebra)," Invent. Math., vol. 113, no. 1, pp. 41-55, 1993, doi: 10.1007/BF01244301.

[15] G. Lyubeznik, "F-modules: applications to local cohomology modules and D-modules in characteristic $p>$ 0," J. Reine Angew. Math., vol. 491, pp. 65-130, 1997, doi: 10.1515/crll.1997.491.65.

[16] G. Lyubeznik, "Finiteness properties of local cohomology modules for regular local rings of mixed chartacteristic: the unramified case," Comm. Algebra, vol. 28, no. 12, pp. 5867-5882, 2000, doi: 10.1080/00927870008827193.

[17] H. Matsumura, Commutative ring theory, paperback ed., ser. Cambridge Studies in Advanced Mathematics. Cambridge, UK: Cambridge University Press, 1989, vol. 8.

[18] L. Melkersson, "Modules cofinite with respect to an ideal," J. Algebra, vol. 285, no. 2, pp. 649668, 2005, doi: 10.1016/j.jalgebra.2004.08.037.

[19] L. Melkersson, "Cofiniteness with respect to ideals of dimension one," J. Algebra, vol. 372, pp. 459-462, 2012, doi: 10.1016/j.jalgebra.2012.10.005.

[20] H. Zöschinger, "Minimax moduln,” J. Algebra, vol. 102, pp. 1-32, 1986.

[21] H. Zöschinger, "Über die maximalbedingung für radikalvolle untermoduln," Hokkaido Math. J., vol. 17, no. 1, pp. 101-116, 1988.

Author's address

Yavar Irani

Islamic Azad University Meshkin-Shahr branch, Department of Mathematics, P.O. Box 5661645936, Ardabil, Iran

E-mail address: yavarir@ymail.com 